\title{
Epidemiología de la enfermedad de Chagas en el estado de Veracruz
}

Grupo de Estudio sobre la Enfermedad de Chagas*,(1) Elsa L Segura, PhD, ${ }^{(2)}$ Alejandro Escobar-Mesa, MC.(3)

\section{Grupo de Estudio sobre la Enfermedad de Chagas, Segura EL, Escobar-MesaA. Epidemiología de la enfermedad de Chagas en el estado deVeracruz. Salud Publica Mex 2005;47:201-208.}

\section{Resumen}

Objetivo. Identificar la seroprevalencia de enfermedad de Chagas, los factores de riesgo de la vivienda e índices entomológicos, para proponer medidas de control en 11 jurisdicciones sanitarias del estado de Veracruz. Material y métodos Entre 1997 y 2001 se hizo un estudio transversal cuya muestra quedó integrada por 281 localidades, 2526 viviendas y 9782 individuos. Se aplicó un cuestionario sobre factores de riesgo, se tomó sangre en papel filtro y se buscaron triatominos en el intra y peridomicilio. Se obtuvo la prevalencia de exposición a factores de riesgo y de seropositividad para la enfermedad. Se hizo análisis bivariado mediante la razón de momios,ii de Mantel y Haenszel e intervalo de confianza a 95\% como prueba de significancia estadística. El análisis multivariado se hizo mediante la regresión logística no condicional y se incluyeron las variables que durante el análisis bivariado mostraron un valor de $p$ hasta de 0.20. El impacto potencial se estimó con base en la fracción etiológica en expuestos. Resultados La prevalencia de enfermedad de Chagas fluctuó entre 0 y $2.8 \%$. Las jurisdicciones con mayor riesgo fueron Tuxpan, Pánuco y Córdoba, y sin riesgo, 0 rizaba. Los principales facto res de riesgo de la vivienda fueron el techo y muro de palma/zacate y piso de tierra, así como la presencia del vector y la ventilación. Conclusiones Es necesario realizar vigilancia epidemioló gica basada en educación para la salud, mejoramiento de la vivienda y uso de insecticidas.

Palabras clave: enfermedad de Chagas/epidemiología; México

\author{
Study Group on Chagas Disease, \\ Segura EL, Escobar-MesaA. \\ Epidemiology of Chagas disease \\ in the state of Veracruz. \\ Salud Publica Mex 2005;47:201-208.
}

\begin{abstract}
A bstract
Objective. To assess the seroprevalence, household risk factors, and entomological indicators, in order to frame control measures in 11 Sanitary Jurisdictions of the state. Material and Methods This study included 281 towns, 2526 households, and 9782 individuals. Data were collected using a questionnaire. Blood was obtained in filter paper and a search for triatomines was conducted inside of and around dwellings. Prevalence rates were used to quantify exposure to risk factors and seropositivity. 0 dds ratios with $95 \%$ confidence intervals and Mantel-H aenszel chi-squared tests were obtained. Multivariate analysis was performed with unconditional logistic regression, variables included in the model were those that had a $p$-value up to 0.20 in the bivariate analysis. The etiologic fraction in the exposed was also obtained. Results. The prevalence of Chagas disease was between 0 and 2.8\%. Jurisdictions at a higher risk were Tuxpan, Panuco and Cordo ba; 0 rizaba showed no risk. The main household risk factors were palma/zacate (palmtree, grass leaves) roof and walls, dirt floor, the presence of the vector, and ventilation. Conclusions. Epidemiological surveillance should emphasize health education, housing improvement, and use of insecticides.
\end{abstract}

Key words: Chagas disease/epidemiology; Mexico

* Paz María Salazar-Schettino, PhD, Gloria Elena Rojas-W astavino, M en C, Margarita Cabrera-Bravo, M en C, Martha Irene Bucio-Torres, M en C, Yolanda Guevara-Gómez, M en C, G uadalupe Silvia García-de la Torre, M en C.

(1) Departamento de Microbiología y Parasitología, Facultad de Medicina. Universidad N acional Autónoma de México, (UN AM). México, DF, México.

(2) Instituto Dr. Mario Fatala Chabén. Buenos Aires,Argentina.

(3) Servicios de Salud de Veracruz.Veracruz,Veracruz, México.

Fecha de recibido: 14 de junio de 2004 • Fecha de aprobado: 30 de marzo de 2005

Solicitud de sobretiros: D ra. Paz María Salazar-Schettino. D epartamento de Microbiología y Parasitología. Facultad de Medicina, Universidad N acional Autónoma de México. Circuito Universitario, Edificio A, segundo piso, Ciudad Universitaria, San Angel, 04510 México, DF, México. Correo electrónico:pazmar@ servidor.unam.mx 
D esde el descubrimiento de Triatoma dimidiata en 1928 por Hoffman, en Veracruz, y los primeros reportes de Mazzotti, en 1936 y 1940,,$^{1-3}$ del vector infectado y de infección aguda en el humano, hasta la fecha han transcurrido varias décadas en que la importancia de la enfermedad de Chagas en México no tuvo su justa valoración. El país tiene las características geográficas, climáticas y socioculturales, y un gran número de transmisores existentes ${ }^{4-6}$ como para haberse considerado su importancia desde el hallazgo de los primeros triatominos naturalmente infectados con Trypanosoma cruzi y, desde luego, con los reportes de casos humanos que no han dejado de publicarse en la literatura correspondiente. Se han hecho estudios seroepidemiológicos en diferentes estados de la República utilizando diferentes antígenos y diferentes pruebas serológicas, por lo que los resultados son difíciles de comparar. ${ }^{7-24}$ Por contar con las condiciones socioeconómicas, climatológicas y ecológicas adecuadas y diferentes en cada jurisdicción sanitaria para permitir la transmisión activa de la enfermedad, y por el interés que las autoridades de la Secretaría de Salud del estado mostraron al respecto, Veracruz se convirtió en el lugar idóneo para el desarrollo de esta investigación. El objetivo del estudio fue identificar la prevalencia de la enfermedad de Chagas en cada jurisdicción sanitaria, los principales factores de riesgo asociados a ella y los índices entomológicos.

\section{Material y métodos}

Estudio epidemiológico transversal hecho entre 1997 y 2001, en 11 jurisdicciones sanitarias del estado de Veracruz, México. Se obtuvo el tamaño de muestra para cada jurisdicción sanitaria a partir de la fórmula para la estimación de una proporción poblacional, ${ }^{25}$ suponiendo una prevalencia de 15\% (con base en las encontradas en otros estudios ${ }^{15-20} \mathrm{y}$ en la experiencia del grupo de trabajo), un nivel de confianza de $95 \%$ y una precisión de $2 \%$.

La unidad última de muestreo fueron todos los individuos que cumplieron los criterios de selección (haber habitado en la comunidad por lo menos durante el último año y no haber sido transfundidos alguna vez en su vida) y que habitaban la vivienda seleccionada mediante muestreo aleatorio irrestricto. Con base en el método de asignación proporcional de la muestra se atribuyó el número de individuos (suponiendo un índice de hacinamiento promedio en el estado de cinco personas por vivienda) con los que contribuiría cada una de las comunidades de menos de 500 habitantes elegidas.
Se decidió trabajar con comunidades pequeñas (de menos de 500 habitantes), debido a que, tal como se ha observado en distintos estudios, incluyendo la Encuesta Nacional Seroepidemiológica de $1987,{ }^{15}$ los focos de transmisión para Chagas están principalmente en localidades rurales y de difícil acceso, características que no necesariamente cumplen las localidades urbanas o semiurbanas.

Se clasificó la vivienda según el material de construcción del techo, muro y piso respecto al riesgo de albergar triatominos. Así, si la vivienda tenía tanto techo como muro y piso de un material clasificado como "sin riesgo" (cemento, concreto, lámina de asbesto o zinc, ladrillo, block, piedra) se designó como "casa"; cuando tanto el techo como el muro y el piso eran de materiales considerados "con riesgo" (madera, corteza, carrizo, bambú, palma, zacate, teja, lámina de cartón, adobe, barro, tierra) se categorizó como "choza," y cuando el techo, el muro o el piso fueron de riesgo, se clasificó como "jacal".

Para el estudio serológico se hizo un tamizaje con las técnicas de hemaglutinación indirecta (HAI) y ELISA en microplaca con eluidos séricos de muestras obtenidas por punción digital, en papel filtro Whatman No. 1, para lo cual se capacitó al personal de salud de cada jurisdicción sanitaria. Se realizaron las técnicas de HAI (considerada positiva con título igual o mayor de 1:16 y negativa con título igual o menor de 1:8) y ELISA (considerada positiva con valores iguales o mayores de 0.200 de densidad óptica -DO-). A todas aquellas personas que resultaron positivas a una o dos pruebas en el tamizaje en papel filtro, se les tomó muestra obtenida por punción venosa periférica para la confirmación en suero con HAI, ELISA e inmunofluorescencia indirecta -IFI- (la cual se considera positiva con título igual o mayor a 1:32), como se recomienda internacionalmente por los centros de referencia de la Organización Panamericana de la Salud (OPS) y la Organización Mundial de la Salud (OMS ${ }^{26}$ para la confirmación diagnóstica de infección con Trypanosoma cruzi. Las tres técnicas se realizaron con antígenos extraídos de una cepa de Trypanosoma cruzi de origen mexicano, con metodologías previamente estandarizadas en nuestro laboratorio. Para la técnica de HAI se prepararon los antígenos de acuerdo con la metodología descrita y empleada en el Instituto Nacional de Chagas "Dr. Mario Fatala Chabén" de la República de Argentina. ${ }^{26}$ El procedimiento se hizo en microplaca de 96 pozos con fondo en $U$; las muestras se procesaron en las diluciones de 1:16, 1:32 y 1:64. La técnica de ELISA se realizó según el método descrito en 1975 por Voller, ${ }^{27}$ con el empleo de un antígeno previamente 
caracterizado, ${ }^{28}$ la técnica de IFI, según el método descrito previamente en el Centro para el Control de Enfermedades (CDC) de Atlanta, Georgia, EUA. ${ }^{29}$

A las personas estudiadas se les clasificó con base en criterios serológicos y clínicos como "No casos" si resultaron negativos a 2 o 3 de las pruebas serológicas y no tenían manifestaciones clínicas de alteración cardiaca; "Infectados" cuando resultaron positivos a 2 o 3 de las pruebas serológicas y no tenían manifestaciones clínicas de alteración cardiaca, y "Enfermos" a quienes fueron positivos a 2 o 3 de las tres pruebas serológicas y tenían manifestaciones clínicas de alteración cardiaca.

En cada vivienda estudiada se emprendió la búsqueda intencionada de triatominos, en el intradomicilio y peridomicilio mediante el método hora/hombre, para integrar los índices entomológicos con el fin de contar con una aproximación del riesgo de infectarse. De los índices sugeridos por Silveira ${ }^{30}$ son tres los más importantes, ya que el riesgo de infección por T. cruzi se relaciona con la presencia del vector en la vivienda (índice de infestación), con la colonización en el interior del domicilio (índice de colonización), y que además el triatomino se encuentre infectado por T. cruzi (índice de infección natural).

Asimismo, se solicitó información sobre edad, sexo, peso, talla, ocupación, escolaridad, lugar de nacimiento, tiempo de residencia en la localidad, lugar de residencia anterior, material de construcción de techos, muros y piso, presencia de fisuras, iluminación y ventilación, número de dormitorios, conocimiento del vector, presencia de triatominos dentro y fuera de la vivienda, hacinamiento, convivencia con animales, realización de fumigaciones, consumo de alcohol y manifestaciones clínicas.

La escolaridad se evaluó a partir de los mayores de 11 años, ya que los menores se encuentran estudiando y, por otro lado, el tener su primaria incompleta o ser "analfabeta" no refleja su grado de escolaridad real.

A cada persona se le explicó con detalle el objetivo de la investigación y de la toma de muestras de sangre y se les pidió la firma de la carta de consentimiento informado. En el caso de los menores de edad, dicho consentimiento fue firmado por el padre, madre o tutor.

Respecto al manejo estadístico, se utilizó una descripción de la población de estudio a partir del porcentaje de las variables cualitativas y se obtuvo la prevalencia de exposición a los factores de riesgo, así como la prevalencia de seropositividad para la enfermedad de Chagas. Para el análisis bivariado se tomó por cada "caso" (individuo infectado o enfermo), a cuatro "no casos" (razón 1:4), que fueran del mismo sexo y \pm 2 años de la edad del caso, esto debido a que la razón caso:no caso hubiera sido de 1:154; y con base en ello, se midió la magnitud de la asociación de los factores considerados de riesgo a través de la razón de momios para la prevalencia, y se evalúo la significancia estadística mediante la Ji de Mantel y Haenszel e intervalos de confianza a $95 \%$. El análisis multivariado se realizó mediante la regresión logística no condicional, con el programa SPSS v.10, y se incluyeron en él aquellas variables que durante el análisis bivariado mostraron un valor de $p$ hasta de 0.20 . El impacto potencial se estimó con base en la fracción etiológica en expuestos.

\section{Resultados}

Se estudió un total de 9782 individuos, provenientes de 9 de las 11 jurisdicciones sanitarias del estado. En dos de ellas no fue posible obtener información, por lo que quedaron fuera de este estudio.

Del tamizaje realizado con eluidos obtenidos de muestras sanguíneas en papel filtro de los individuos estudiados (9 782), resultaron positivos a una o dos pruebas 624 y sólo se realizó confirmación en suero a 392 , de los cuales 63 fueron positivos a 2 o 3 de las pruebas realizadas; se observó que hubo 33 personas positivas a tres pruebas, 21 positivas a HAI y ELISA, y nueve positivos a ELISA e IFI.

Con base en lo anterior se obtuvo una prevalencia de enfermedad de Chagas, que fluctuó desde $0 \%$ (Martínez de la Torre, Orizaba y Coatzacoalcos), hasta 2.8\% (Tuxpan) (cuadro I); no hubo diferencias estadísticamente significativas $(p>0.05)$ por sexo en ninguna de las jurisdicciones sanitarias estudiadas.

$\mathrm{Al}$ evaluar por grupos de edad las jurisdicciones positivas, se observó que la infección se presentó principalmente en los menores de 18 años de edad en las Jurisdicciones Sanitarias de Pánuco, Tuxpan, Xalapa y San Andrés Tuxtla (cuadro II).

Respecto de los factores que han sido considerados como de riesgo para la permanencia de los transmisores y, por lo tanto, para la interacción del huésped potencial y el vector, se encontró que en la mayoría de las jurisdicciones la población en estudio convive con perros y con gatos, mismos que pueden servir como fuente de alimentación para los triatominos y de esta manera facilitar la interacción huésped-vector; sin embargo, la mayoría de los animales domésticos suelen dormir en el patio. El principal tipo de vivienda es la choza, con presencia de fisuras (lugar en que los triatominos suelen habitar); aunque en su mayoría expresaron haber visto salir triatominos de dichas fisuras, la ventilación e iluminación de las viviendas era in- 
Cuadro I

Prevalencia de la enfermedad de Chagas por jurisdicción sanitaria y sexo, en La población estudiada del estado de Veracruz, 1997-2001

\begin{tabular}{|c|c|c|c|c|c|c|c|c|c|}
\hline \multirow[b]{2}{*}{ Jurisdicción sanitaria } & \multicolumn{3}{|c|}{ Hombres* } & \multicolumn{3}{|c|}{ Mujeres } & \multicolumn{3}{|c|}{ Total } \\
\hline & $\mathrm{N}$ & $(t)$ & $\%$ & $\mathrm{~N}$ & $(+)$ & $\%$ & $\mathrm{~N}$ & $(+)$ & $\%$ \\
\hline Pánuco & 582 & 13 & 2.2 & 653 & 7 & 1.0 & 1235 & 20 & 1.6 \\
\hline Tuxpan & 320 & 9 & 2.8 & 446 & 14 & 3.1 & 766 & 23 & 2.8 \\
\hline Poza rica & - & - & - & - & - & - & 1182 & - & - \\
\hline Martínez de la Torre & 603 & 0 & 0 & 650 & 0 & 0 & 1253 & 0 & 0 \\
\hline Jalapa & 480 & 1 & 0.2 & 803 & 5 & 0.6 & 1283 & 6 & 0.5 \\
\hline Córdoba & 379 & 5 & 1.3 & 378 & 5 & 1.3 & 757 & 10 & 1.3 \\
\hline Orizaba & 329 & 0 & 0 & 325 & 0 & 0 & 654 & 0 & 0 \\
\hline Veracruz & 551 & 2 & 0.3 & 685 & 0 & 0 & 1236 & 2 & 0.2 \\
\hline Cosamaloapan & - & - & - & - & - & - & 1271 & - & - \\
\hline San Andrés Tuxtla & 558 & 1 & 0.17 & 721 & 1 & 1.13 & 1279 & 2 & 0.1 \\
\hline Coatzacoalcos & 583 & 0 & 0 & 736 & 0 & 0 & 1319 & 0 & 0 \\
\hline$* p>0.05$ & & & & & & & & & \\
\hline
\end{tabular}

Cuadro II

DistribuCión POR EDAD DE LOS INFECTADOS Y ENFERMOS, EN SEIS JURISDICCIONES SANITARIAS en las que Se detectaron Casos de enfermedad de Chagas. Veracruz, México, 1997-2001

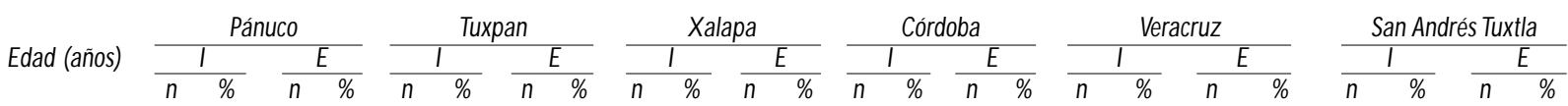

\begin{tabular}{lrrrrrrrrrrrrrrrrrrrrrrrrrrrr}
1 a 4 & - & - & - & - & 1 & 5.0 & - & - & & & & 1 & 10.0 & & - & - & - & - & - & - & - & - \\
\hline 5 a 14 & 2 & 10.0 & - & - & 4 & 19.0 & - & - & - & - & - & - & 2 & 20.0 & - & - & - & - & - & - & 1 & 100 & - & - \\
\hline 15 a 18 & 1 & 5.0 & - & - & 1 & 5.0 & - & - & - & - & - & - & - & - & - & - & 1 & 50.0 & - & - & - & - & - & - \\
\hline 19 a 34 & 4 & 20.0 & - & - & 5 & 24.0 & - & - & 2 & 50.0 & 2 & 100 & 3 & 30.0 & - & - & 1 & 50.0 & - & - & - & - & - & - \\
\hline 35 a 64 & 10 & 50.0 & - & - & 7 & 33.0 & 2 & 100 & 1 & 25.0 & - & - & 3 & 30.0 & - & - & - & - & - & - & - & - & - & - \\
\hline 65 y más & 3 & 15.0 & - & - & 3 & 14.0 & - & - & 1 & 25.0 & - & - & 1 & 10.0 & - & - & - & - & - & - & - & - & 1 & 100 \\
\hline Total & 20 & 100 & - & - & 21 & 100 & 2 & 100 & 4 & 100 & 2 & 100 & 10 & 100 & - & - & 2 & 100 & - & - & 1 & 100 & 1 & 100
\end{tabular}

$\mathrm{I}=$ infectado

$\mathrm{E}=$ enfermo

adecuada, (factores que representan características deseables para la colonización por parte del vector), el cual, además de poder alimentarse de la sangre de los animales domésticos, puede hacerlo también de las personas que habitan las viviendas ya que en un mayor número de éstas existe presencia de hacinamiento (lo que significa que más de tres personas duermen en una misma habitación), aunado a esto, tampoco se acostumbra rociar insecticida (cuadro III).
El único transmisor domiciliado fue Triatoma dimidiata, el cual se encontró en los siguientes porcentajes: intradomiciliado $89 \%$, y peridomiciliado $11 \%$. Los ecotopos fueron: dormitorio $84 \%$, bodegas $3 \%$, otros $13 \%$. La distribución en el dormitorio fue en el murocama de $56 \%$, cama $39 \%$, piso $4 \%$, ropero y ventana $1 \%$. El resultado del cálculo de los índices entomológicos en forma global para el estado fue $13.5 \%$ para el índice de infestación, lo que significa que de todas las 
Cuadro III

Prevalencia de factores de riesgo para enfermedad de Chagas en la población estudiada. VeracruZ, MÉxico, 1997-2001

Factor de Riesgo

No. $\quad \%$

Convivencia con perro

\begin{tabular}{lll} 
Sí & 7056 & 72.1 \\
\hline No & 2726 & 27.9
\end{tabular}

Convivencia con gato

\begin{tabular}{lll} 
Sí & 4636 & 47.3 \\
\hline No & 5146 & 52.6
\end{tabular}

Lugar donde duermen los animales

\begin{tabular}{lrr} 
Dentro de la casa & 572 & 5.8 \\
\hline Establo & 36 & 0.4 \\
\hline Patio & 7325 & 74.8 \\
\hline O tro & 1849 & 18.9
\end{tabular}

Tipo de vivienda

\begin{tabular}{lrr} 
Choza & 4891 & 50.0 \\
\hline Jacal & 4668 & 47.7 \\
\hline Casa & 223 & 2.2
\end{tabular}

Fisuras

\begin{tabular}{lll} 
Sí & 8059 & 82.4 \\
\hline No & 1723 & 17.6
\end{tabular}

Haber visto salir triatominos de las fisuras

\begin{tabular}{lll} 
Sí & 2606 & 26.6 \\
\hline No & 7176 & 73.4
\end{tabular}

Ventilación

\begin{tabular}{lll} 
Mala & 2695 & 27.6 \\
\hline Regular & 3851 & 39.3 \\
\hline Buena & 3236 & 33.1
\end{tabular}

Iluminación

\begin{tabular}{lll} 
Mala & 1498 & 15.3 \\
\hline Regular & 4282 & 43.8 \\
\hline Buena & 4002 & 40.9
\end{tabular}

Hacinamiento

\begin{tabular}{lll} 
Sí & 5230 & 53.5 \\
\hline No & 4552 & 46.5
\end{tabular}

Dormir junto a la pared

\begin{tabular}{lll} 
Sí & 8267 & 84.5 \\
\hline No & 1515 & 15.5
\end{tabular}

Fumigación

\begin{tabular}{lll} 
Sí & 4399 & 44.9 \\
\hline No & 5383 & 55.1
\end{tabular}

viviendas examinadas, en dicho porcentaje se encontraron triatominos. Para el índice de colonización el resultado fue $60.8 \%$, esto implica que entre los domicilios con triatominos, en tal porcentaje había diferentes estadios del ciclo biológico del transmisor. El índice de infección natural fue $10.6 \%$, es decir, que de cada 100 especímenes capturados, aproximadamente 11 estuvieron infectados con T. cruzi.

En el análisis bivariado se encontró que los factores potencialmente asociados fueron: el sexo (femenino), la escolaridad baja (primaria incompleta o menos), el que duerman dentro de la vivienda los animales domésticos, la ventilación e iluminación inadecuadas, el conocer al vector, y haberlo visto dentro de la vivienda saliendo de las fisuras (cuadro IV). Estas variables fueron incluidas en el análisis multivariado; finalmente,

$$
\text { Cuadro IV }
$$

\section{Factores asociados a la enfermedad de Chagas. Veracruz, México, 1997-2001}
Variable Casos No casos RMPc* IC $95 \% \quad P$

Sexo

\begin{tabular}{llllll} 
Femenino & 32 & 151 & 0.69 & $0.38-1.25$ & 0.18 \\
\hline Masculino & 31 & 101 & 1.00 & &
\end{tabular}

Escolaridad

0

\begin{tabular}{lrrrrr} 
Primaria incompleta o menos & 56 & 203 & 3.22 & $090-13.65$ & 0.07 \\
\hline Primaria completa o más & 3 & 35 & 1.00 & &
\end{tabular}

Dormitorio de animales

\begin{tabular}{lrrrrr} 
Dentro de la vivienda & 5 & 10 & 2.09 & $0.59-6.98$ & 0.19 \\
\hline Fuera de la vivienda & 58 & 242 & 1.00 & &
\end{tabular}

Fuera de la vivienda

Ventilación

\begin{tabular}{llllll} 
Inadecuada & 42 & 142 & 1.55 & $0.84-2.08$ & 0.17 \\
\hline Adecuada & 21 & 110 & 1.00 & &
\end{tabular}

lluminación

\begin{tabular}{llllll} 
Inadecuada & 42 & 140 & 1.60 & $0.86-2.98$ & 0.11 \\
\hline Adecuada & 21 & 112 & 1.00 & &
\end{tabular}

Conocer al vector

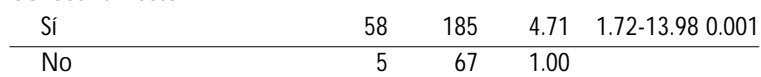

Haberlas visto dentro de la vivienda

\begin{tabular}{lrrrrr} 
Sí & 44 & 124 & 2.18 & $1.08-4.46$ & 0.01 \\
\hline N 0 & 14 & 86 & 1.00 & &
\end{tabular}

Salir el vector de las fisuras

\begin{tabular}{llllll} 
Sí & 36 & 101 & 1.99 & $1.10-3.62$ & 0.01 \\
\hline No & 27 & 151 & 1.00 & &
\end{tabular}

* Razón de momios para la prevalencia cruda 
quedó un modelo que incluyó a sólo dos de ellas: ventilación inadecuada y conocer al vector (cuadro V).

\section{Discusión}

Hasta ahora, los estudios sobre seroprevalencia en el estado de Veracruz han omitido los factores de riesgo asociados a la enfermedad de Chagas: este es el primer estudio en el que se relacionan ambos. Los datos sobre seroprevalencia en el mismo han sido de $3 \%,{ }^{15}$ $1 \%$ en donantes ${ }^{31}$ y $4 \%$ en grupos de donadores provenientes de todo el país, concentrados en el Hospital General de México. ${ }^{32}$

En este estudio, llevado a cabo en nueve de las 11 jurisdicciones sanitarias, las prevalencias fluctuaron entre 0 y $2.8 \%$ en seis jurisdicciones donde hubo individuos positivos a 2 o 3, de las tres pruebas serológicas propuestas por la OMS para detectar anticuerpos anti T. cruzi, ${ }^{33}$ lo que difiere respecto a los estudios antes señalados debido a que el tipo de muestreo fue aleatorio entre las comunidades menores de 500 habitantes y no elegidas mediante muestreo por conveniencia, y se dirigió a comunidades que se saben infestadas por triatominos o con presencia de casos de infección. Las prevalencias más altas se presentaron en las jurisdicciones ubicadas en el norte del estado: $1.6 \%$ en Pánuco y $2.8 \%$ en Tuxpan. Estas jurisdicciones sanitarias también fueron las que presentaron los índices de infestación más elevados, 30 y $34.5 \%$, respectivamente, mientras que fue de $13.5 \%$ para todo el estado; los índices de colonización de ambas jurisdicciones sanitarias fueron de 62 y $67 \%$, respectivamente, porcentaje mayor al del ámbito estatal que fue de $60.8 \%$, lo que indica que el ciclo biológico del transmisor se está llevando a cabo dentro de la vivienda; de igual manera los ejemplares capturados presentaron 8 y $19 \%$ de infección con T. cruzi y en el estado el índice de infección natural global fue de $10.6 \%$. Los índices entomológicos que se correlacionaron estrechamente a la seropositividad de las Jurisdicciones Sanitarias Pánuco y Tuxpan fueron el de infestación y el de infección natural.

Cuadro V

Factores de riesgo para enfermedad de Chagas en el estado de Veracruz, México, 1997-2001

\begin{tabular}{lccc} 
Variable & RM P* & IC 95\% & P \\
Ventilación inadecuada & 1.56 & $1.10-2.85$ & 0.04 \\
\hline Conocer al vector & 3.68 & $1.40-9.71$ & 0.008 \\
* Razón de momios ajustada para la Prevalencia & &
\end{tabular}

La distribución por grupos de edad mostró que en prácticamente todas las jurisdicciones los jóvenes constituyen alrededor de la mitad de la población. La relación por sexo no mostró cifras estadísticamente significativas en los individuos estudiados.

Del número total de infectados o enfermos en las jurisdicciones hubo varios casos que se presentaron en menores de 18 años de edad; así, tenemos a tres infectados en Pánuco $(15 \%)$, seis en Tuxpan $(26 \%)$, tres en Córdoba (30\%), una persona en Veracruz (50\%) y una en San Andrés Tuxtla (50\%), lo cual indica que la infección se está trasmitiendo en forma activa en estas jurisdicciones. Tuxpan, específicamente, es un sitio en el que existe población afectada en edad prescolar, escolar, adolescentes y adultos. También se observa que, como era de esperarse, la enfermedad (es decir con manifestaciones clínicas de alteración cardiaca), fue más frecuente en la población económicamente activa (principalmente de 19 a 64 años de edad). La transmisión activa es un indicador que puede servir como referencia para la aplicación de medidas de control y, de repetirse el estudio dentro de cinco años sobre seroprevalencia en las jurisdicciones en que se encontraron casos, se comprobaría la eficacia de tales medidas.

Factores que en otros estudios han sido considerados de riesgo, como el realizado en varias localidades del estado de Puebla, ${ }^{34}$ también se manifiestaron en el estado. La escolaridad es un factor que se asocia al grado de "cultura médica" de una población, pues se considera que a mayor escolaridad, mayor probabilidad de tener información para el autocuidado del estado de salud, del entorno, y viceversa. Al respecto, en todas las jurisdicciones se encontró que la mayoría de las personas sabe leer y escribir, pero sin haber acudido formalmente a la escuela, excepto en Córdoba, donde la mayoría de los individuos tenía como grado máximo de escolaridad la primaria incompleta. En todas las jurisdicciones la ocupación de los hombres mayores de 15 años de edad, fue, principalmente, la agricultura, mientras que las mujeres se dedican sobre todo al hogar.

Algunos factores facilitan la infestación y permanencia de los triatominos en las viviendas y, por lo tanto, la interrelación huésped-vector. Uno de ellos es la convivencia con perros y gatos que representan una fuente de alimentación para los triatomas; en todas las jurisdicciones sanitarias la convivencia con estos animales fluctuó entre 47 y $72 \%$ de la población. El material de construcción de la vivienda representa un factor importante en la transmisión de la enfermedad, ya que puede proveer al vector las características para la permanencia y su reproducción en ella. Se observó que los materiales de los techos de las viviendas fueron 
principalmente la lámina de zinc, carrizo/bambú, teja o lámina de cartón. Para los muros, los materiales principales fueron el barro o la madera y en el piso, la tierra. Todos estos materiales pueden facilitar la colonización y reproducción del vector. En todas las jurisdicciones las viviendas se clasificaron principalmente como "choza" (Pánuco, Martínez de la Torre, Xalapa y Veracruz), o como "jacal" (Tuxpan, Córdoba, Orizaba, San Andrés Tuxtla y Coatzacoalcos). En todas ellas había fisuras en techo, muro y piso. En general, la iluminación y la ventilación de la vivienda eran malos o regulares, hubo hacinamiento y las personas dormían principalmente junto a la pared, facilitando con ello la posibilidad de alimentación a los vectores.

Con base en los hallazgos del análisis de riesgo, es recomendable y evidente que debe mejorarse la vivienda, especialmente material de construcción; asimismo, la ventilación inadecuada podría mejorarse si se recomienda la redistribución de puertas y ventanas de modo que la penetración del aire pueda ser fluida; se estima que si se eliminara la ventilación inadecuada disminuirían $36 \%$ de los casos. Con medidas como éstas se ha logrado el control de los transmisores. ${ }^{35,36}$ Deberán desarrollarse acciones para disminuir la colonización de estos vectores, principalmente fumigaciones periódicas con insecticidas que tengan efecto residual, así como promover el reconocimiento e identificación del vector entre la comunidad, como parte de la fauna nociva, mediante un programa de difusión masiva que recomiende las medidas básicas de control de este problema: higiene de la vivienda, mejoramiento de la vivienda y fumigaciones periódicas. Debido a que el conocimiento del vector fue un factor de riesgo asociado a la infección por T. cruzi, si en el programa de control del transmisor se promueven de manera enfática las medidas para eliminarlo o controlarlo, se evitarían $73 \%$ de los casos de enfermedad de Chagas.

\section{Agradecimientos}

A los doctores Edith Rodríguez y Mauro Loyo, y al ingeniero Leopoldo Hidalgo, de los Servicios de Salud del estado de Veracruz, por su apoyo en el trabajo de campo. A la doctora Adela Ruiz Hernández, por su colaboración en el proceso de las pruebas serológicas.

\section{Referencias}

1. Hoffmann C. N ota acerca de un probable transmisor de la trypanosomiasis humana en el estado de Veracruz. Rev Mex Biol 1928;8:12-18.
2. Mazzotti L. Investigación sobre la existencia de la enfermedad de Chagas en el país. Demostración de los tripanosomas en los reduvídeos transmisores. Med Mex 1936;282(16):584-585.

3. Mazzotti L. Dos casos de enfermedad de Chagas en el Estado de 0 axaca. Gac Med Mex 1940;70:417-420.

4. Lent $H, W$ ygodzinsky P. Revision of triatominae (Hemiptera, Reduvidae) and their signicance as vector of Chagas disease. Bull Am Mus $\mathrm{N}$ at $\mathrm{H}$ ist 1979;163:124-520.

5. Salazar-Schettino PM, De Haro I, U ribarren T. Chagas disease in Mexico. Parasitology Today 1988:4(12):348-352.

6. C arcavallo R, Galíndez-Girón J, Jurberg J, Galvão C, Lent H. Geographical distribution and alti-latitudinal dispersion. En: Carcavallo $R$, Galíndez-Girón J, Jurberg J, Lent $H$, Eds. Atlas of C hagas disease vectors in the Americas. Rio de Janeiro: Editora Fiocruz; 1999: vol.III; 747-792. 7. Salazar-Schettino PM, C astrejón J, Rodríguez H,Tay J. Miocarditis chagásica crónica en México.Tercer caso comprobado por exámenes parasitoscópicos. Prensa Med Mex 1979;5-6:115-120.

8. Salazar-Schettino PM, Tay J, O ntiveros A, Jiménez J, De Haro I, Bucio M et al. Enfermedad de Chagas en México. Presentación de casos clínicos. Rev Fac Med 1983;25:77-82.

9. Salazar-Schettino PM, Tay J, Bucio M, De Haro I,Anzures ME, FloresAyala S. Primer caso de megaesófago con serología positiva a Trypanosoma cruzi. Salud Publica Mex 1984;26:452-455.

10. Salazar-Schettino PM, D e Haro I, Sánchez-Vega J, C anepa D. Reporte de tres casos de enfermedad de Chagas en fase crónica. Rev Mex Patol Clin 1986:33:55-63.

11. Salazar-Schettino PM, Barrera M, Bucio M.Transmisión de Trypanosoma cruzi por transfusión sanguínea. Primer caso humano en México. Rev Mex Patol Clin 1989; 36:57-59.

12. Becerra I, Manrique M, Padrón A, 0 rtiz G. Megaesófago en la enfermedad de Chagas. Rev Hosp Juarez 2001; 68(1):31-36. 13. Cordero P, Zárate R, Ramos C, Cordero C. Miocardiopatía dilatada chagásica en el estado de Chiapas, México. Rev Mex Cardiol 2002;4:153-157.

14. Tay J, Salazar-Schettino PM, 0 ntiveros A, Jiménez J, De Haro I, G arcía $Y$ et al. Epidemiologic study of $C$ hagas'disease in a town in 0 axaca, Mexico. Bull PAHO 1986; 20(4):358-365.

15.Velasco-C astrejón O,Valdespino JL,Tapia-C onyer R, Salvatierra B, Guzmán-Bracho C, Magos $C$ et al. Seroepidemiología de la enfermedad de Chagas en México. Salud Publica Mex 1992; 34:186-196.

16. Biagi F,Tay J, G uzmán G, Fong P.Tetitlán Guerrero foco endémico de enfermedad de Chagas. Rev Fac Med 1964; 6(9):625-631.

17. Q uintal E, Zavala J, Rodríguez M. La enfermedad de Chagas en el estado de Yucatán, México. Rev Invest Clin 1975; 27:255-258.

18. Goldsmith $S, K$ agan $G, Z$ árate R, Reyes A, C edeño J. Estudios epidemiológicos de la enfermedad de Chagas en 0 axaca, México. Bol 0 ficina Sanit Panam 1979; 87:1-19.

19. Goldsmith S, 0 rtega $M$, Zárate R, Zárate L, Beltrán $F$. Seroepidemiologic surveys for Chagas' disease in Chiapas, México.Arch Invest Med 1983;

14:43-50.

20. Salazar-Schettino PM, Tay J, Ruiz-Hernández A, D e H aro I, Bucio M, Jiménez J et al. Seropositividad a Trypanosoma cruzi en cuatro grupos de población del estado de 0 axaca. Salud Publica Mex 1984; 26:589-595. 21. Sánchez B. Miocardiopatía crónica e infección por T. cruzi en una localidad de Morelos y Tabasco (tesis). México, DF: Facultad de Medicina, Universidad $\mathrm{N}$ acional Autónoma de México; 1988.

22. Tay J. La enfermedad de Chagas en la República Mexicana. Salud Publica Mex 1980; 22:409-450.

23. Cortés JM,Velasco-C astrejón O, Labastida MH, Melchor AH, D uarte $N$, D e Torre R. La enfermedad de Chagas en Santiago Yosotiche, 0 axaca, México. Salud Publica Mex 1985;27:60-65.

24. Dumonteil E. Update on Chagas' disease in Mexico. Salud Publica Mex 1999; 41:322-327. 
25. Scheaffer RL, Mendenhall W O. Muestreo irrestricto aleatorio. En: Grepe N, ed. Elementos de muestreo. México, DF: Grupo Editorial Iberoamérica; 1987: 321.

26. Instituto N acional de Chagas "Dr. Mario Fatala Chabén". Enfermedad de Chagas y otras parasitosis. Manual de laboratorio. 8 edición Buenos Aires, Argentina: Ministerio de Salud y Acción Social de la República Argentina; 1996.

27.Voller A, D raper C, Bidwell D, Bartlett A. Microplate Enzyme-Linked Immunosorbent Assay for Chagas' D isease. Lancet 1975 February; 22:

426-428.

28. Bucio M, Cabrera M, Segura E, Zenteno E, Salazar-Schettino PM. Identification of immunodominant antigens in Mexican strains of Trypanosoma cruzi. Immunol Invest 1999; 28(4):257-268.

29. Centers for Disease C ontrol, U.S. D epartment of Health, Education and $W$ elfare. $A$ procedural guide to the performance of the indirect fluorescent Antibody test for toxoplasmosis. Atlanta (GA):CDC;1970. $24 \mathrm{p}$.

30. Silveira A, De Rezende D, Correia M. Risk measure of domestic transmission of $C$ hagas disease, through a new entomological indicator. Mem Inst 0 swaldo Cruz 1984;79 Suppl:113-115.
31. Guzmán-Bracho C, García-G arcía C, Floriani-Verdugo I, G uerreroMartínez S,Torres-Cosme M, Ramírez-Melgar $C$ et al. Riesgo de transmisión de Trypanosoma cruzi por transfusión de sangre en México. Rev Panam Salud Publica 1998; 4(2):94-98.

32. Cabrera M, Bucio M, Rojo J, Bonifaz R, GuevaraY, Salazar PM. Detection of antibodies against Trypanosoma cruzi in blood donors in the General Hospital of Mexico City. Rev Patol Trop 2004; 33(1):71-80. 33. Universidad $\mathrm{N}$ acional Autónoma de México. Taller Internacional de Epidemiología, Diagnóstico y Control de la Enfermedad de Chagas. 0 axaca, 0 axaca: UN AM; 2002:14.

34. Sosa-Jurado F, Zumaquero-Ríos JL, Reyes PA, Cruz-García A, Guzmán-Bracho $C$, Monteón VM. Factores bióticos y abióticos que determinan la seroprevalencia de anticuerpos contra Trypanosoma cruzi en el municipio de Palmar de Bravo, Puebla, México. Salud Publica Mex 2004; 46:39-48.

35. Pan American Health O rganization. Iniciativa del C ono Sur. PAHO document. N 0. PN SP/92-18.1::W ashington, DC: Pan A merican Health Organization $1993.36 \mathrm{p}$.

36. Moncayo A. Progress towards interruption of transmission of Chagas disease. Mem Inst 0 swaldo Cruz 1999;94(Suppl 1):401-404. 